Теорія Ймовір. та Матем. Статист. Вип. 77, 2007
Theor. Probability and Math. Statist.

No. 77, 2008, Pages 31-38 S 0094-9000(09)00745-5

Article electronically published on January 14, 2009

\title{
ANALYTICAL PROBLEMS OF THE ASYMPTOTIC BEHAVIOR OF MARKOV FUNCTIONALS. I
}

UDC 519.21

\author{
S. V. DEGTYAR'
}

\begin{abstract}
Some results are given for the asymptotic behavior of Markov functionals of a homogeneous ergodic Markov process.
\end{abstract}

\section{INTRODUCTION}

Let $X(t)$ be a homogeneous Markov ergodic process [1] with either discrete or continuous time, and let $X(t)$ take values in a phase space $(E, \mathcal{B})$. For the sake of simplicity we assume that $E$ is a complete separable metric space, that $\mathcal{B}$ is the $\sigma$-algebra of Borel sets, and that $X(t)$ is a stochastically continuous process in the case of continuous time. The transient probability and invariant probability distribution of the process $X(t)$ are denoted by $P(t, x, A), t \geq 0, x \in E, A \in \mathcal{B}$, and $\pi(A), A \in \mathcal{B}$, respectively.

Consider a family $\xi_{\varepsilon}(t)$ of Markov functionals that depends on a small parameter $\varepsilon>0$ and is asymptotically degenerate. The latter means that

$$
\begin{gathered}
\xi_{\varepsilon}(0)=\xi(0), \quad \varepsilon>0, \\
\lim _{\varepsilon \rightarrow 0} \mathbb{P}_{x, i}\left\{\xi_{\varepsilon}(t) \neq i\right\}=0
\end{gathered}
$$

for all $x \in E, i \in I$, and $t \geq 0$. Here $\mathbb{P}_{x, i}$ is the regular conditional probability given $X(0)=x, \xi(0)=i$.

Conditions (1)-(2) are equivalent to the condition that the joint distribution of the vector $\left\{X(t), \xi_{\varepsilon}(t)\right\}$ converges as $\varepsilon \rightarrow 0$ to the distribution of the vector $\{X(t), \xi(0)\}$.

Below we consider Markov functionals with a countable space of states $I=\{1,2, \ldots\}$. In the case of continuous time, we assume that the trajectories of $\{X(t), \xi(t)\}$ are right continuous and have limits on the left with probability one.

The process $X(t)$ is assumed to be aperiodic in the case of continuous time; that is,

$$
\lim _{t \rightarrow \infty} \int_{E} \varphi(y) P_{t}(x, d y)=\int_{E} \pi(d y) \varphi(y)
$$

for all $x \in E$ and all continuous bounded functions $\varphi(y)$. If the time is discrete, we additionally require that

$$
\lim _{k \rightarrow \infty} P_{k}(x, A)=\pi(A)
$$

for all $x \in E$ uniformly in $A \in \mathcal{B}$.

2000 Mathematics Subject Classification. Primary 60K15, 60J25.

Key words and phrases. Markov functionals, homogeneous ergodic process. 
One can prove that there exists a Markov moment $\tau$ and a set $D \in \mathcal{B}$ such that $\pi(D)>0$ and

$$
\begin{gathered}
\mathbb{P}_{x}\{X(\tau) \in A\}=\pi_{D}(A), \\
\sup _{x \in D}\left[1-\mathbb{P}_{x, i}\left\{\xi_{\varepsilon}(\tau)=i\right\}\right] \underset{\varepsilon \rightarrow 0}{\longrightarrow} 0, \\
\sup _{x \in D} \mathbb{P}(\tau, \tau>T) \underset{T \rightarrow \infty}{\longrightarrow} 0, \\
\tau \geq 1 \quad \mathbb{P}_{x} \text {-almost surely }
\end{gathered}
$$

for all $i \in I$, where $\pi_{D}(A)=\pi(A D) / \pi(D)$.

Put $m=\mathbb{P}_{\pi_{D}, i} \tau$. Here and in what follows,

$$
\mathbb{P}_{\pi_{D}, i}(\cdot)=\int_{D} \pi_{D}(d x) \mathbb{P}_{x, i}(\cdot) .
$$

\section{MAIN RESUlts}

The first result of this section deals with the case of continuous time.

Theorem 1. Let conditions (1), (2), and (3) hold. Assume also that

$$
\sup _{\varepsilon>0} \mathbb{P}_{x, i}\left\{\xi_{\varepsilon}(t)=i, \xi_{\varepsilon}(s) \neq i\right\} \underset{s \rightarrow t}{\longrightarrow} 0
$$

for all $t \geq 0, x \in E$, and $i \in I$. If there exists a matrix $C=\left\|c_{i j}\right\|_{i, j=1}^{\infty}$ such that

$$
\begin{gathered}
\lim _{\varepsilon \rightarrow 0} \frac{1}{\varepsilon m}\left[\mathbb{P}_{\pi_{D}, i}\left\{\xi_{\varepsilon}(\tau)=i\right\}-1\right]=c_{i i}, \\
\lim _{\varepsilon \rightarrow 0} \sum_{j \neq i}\left[\frac{1}{\varepsilon m} \mathbb{P}_{\pi_{D}, i}\left\{\xi_{\varepsilon}(\tau)=j\right\}-c_{i j}\right]=0, \\
\sum_{j} c_{i j}=0, \quad \sup _{i}\left|c_{i i}\right|<\infty,
\end{gathered}
$$

then

$$
\mathbb{P}_{x, i}\left[\varphi(X(t)), \xi_{\varepsilon}(t)=j\right]-p_{i j}(u) \int_{E} \pi(d y) \varphi(y) \underset{\substack{\varepsilon \rightarrow 0 \\ t \rightarrow \infty \\ \varepsilon t \rightarrow u}}{\longrightarrow} 0
$$

for all $x \in E, i \in I$, and all continuous bounded functions $\varphi(y)$ where $p_{i j}(u)$ is the entry $(i, j)$ of the matrix $e^{u C}, C=\left\|c_{i j}\right\|_{i, j=1}^{\infty}$.

Remark 1. Assume that

$$
-\infty<c<c_{i i} \quad \text { for all } i \in I
$$

instead of (12). Put $-c_{i i}=\lambda_{i}$ and $c_{i j}=\lambda_{i} \pi_{i j}$ for $i \neq j$. If $\lambda_{i}>0$, then we let $\pi_{i i}=0$; otherwise, that is, if $\lambda_{i}=0$, we let $\pi_{i j}=0$ for $i \neq j$ and $\pi_{i i}=1$ for all $i$. Then

$$
p_{i j}(u)=\sum_{n=0}^{\infty} p_{i j}^{(n)}(u)
$$

under assumption (13) where

$$
\begin{gathered}
p_{i j}^{0}(u)=\delta^{i j} e^{-\lambda_{i} u} \\
p_{i j}^{(n+1)}(u)=\sum_{k \neq i} \int_{0}^{u} \lambda_{i} e^{-\lambda_{i}(u-s)} \pi_{i k} p_{k j}^{(n)}(s) d s, \quad n=0,1,2, \ldots
\end{gathered}
$$


Proof. Fix a bounded continuous function $\varphi(x) \geq 0$ and a state $l \in\{1,2, \ldots\}$. Let

$$
f_{\varepsilon}^{i}(x, t)=\mathbb{P}_{x, i}\left[\varphi(X(t)), \xi_{\varepsilon}(t)=l\right] .
$$

By the law of total probability,

$$
\begin{aligned}
f_{\varepsilon}^{i}(x, t)= & \mathbb{P}_{x, i}\left[\varphi(X(t)), \xi_{\varepsilon}(t)=l, t<\tau\right] \\
& +\int_{E} \int_{0}^{t} \sum_{j} \mathbb{P}_{x, i}\left\{X(\tau) \in d y, \tau \in d u, \xi_{\varepsilon}(\tau)=j\right\} f_{\varepsilon}^{j}(y, t-u) .
\end{aligned}
$$

For $i \neq j$, put

$$
\begin{gathered}
Q_{\varepsilon}^{i}(x, d y \times d u)=\mathbb{P}_{x, i}\left\{X(\tau) \in d y, \tau \in d u, \xi_{\varepsilon}(\tau)=i\right\}, \\
Q_{\varepsilon}^{i j}(x, d y \times d u)=\mathbb{P}_{x, i}\left\{X(\tau) \in d y, \tau \in d u, \xi_{\varepsilon}(\tau)=j\right\}, \\
g_{\varepsilon}^{i}(x, t)=\mathbb{P}_{x, i}\left[\varphi(X(t)), \xi_{\varepsilon}(t)=l, t<\tau\right] .
\end{gathered}
$$

Now equation (15) can be rewritten as follows

$$
f_{\varepsilon}^{i}(x, t)=g_{\varepsilon}^{i}(x, t)+Q_{\varepsilon}^{i} * f_{\varepsilon}^{i}(x, t)+\sum_{j \neq i} Q_{\varepsilon}^{i j} * f_{\varepsilon}^{j}(x, t) .
$$

This implies that

$$
f_{\varepsilon}^{i}(x, t)=H_{\varepsilon}^{i} * g_{\varepsilon}^{i}(x, t)+\sum_{j \neq i} R_{\varepsilon}^{i j} * f_{\varepsilon}^{j}(x, t),
$$

where $H_{\varepsilon}^{i}(x, d y \times d t)$ is the potential of the kernel $Q_{\varepsilon}^{i}(x, d y \times d t)$, that is,

$$
\begin{gathered}
H_{\varepsilon}^{i}(x, d y \times d t)=\sum_{r=0}^{\infty}\left(Q_{\varepsilon}^{i}\right)^{* r}(x, d y \times d t), \\
R_{\varepsilon}^{i j}(x, d y \times d t)=H_{\varepsilon}^{i} * Q_{\varepsilon}^{i j}(x, d y \times d t), \quad i \neq j .
\end{gathered}
$$

First we consider the case of

$$
\sup _{x \in E} \sum_{j \neq i} R_{\varepsilon}^{i j}(x, E \times[0, \infty)) \leq r<1
$$

for all sufficiently small $\varepsilon>0$ and all $i \in I$.

Put

$$
h_{\varepsilon}^{i}(x, t)=H_{\varepsilon}^{i} * f_{\varepsilon}^{i}(x, t)
$$

and define the sequence of functions $h_{\varepsilon, k}^{i}(x, t)$ by

$$
\begin{gathered}
h_{\varepsilon, 0}^{i}(x, t)=h_{\varepsilon}^{i}(x, t), \\
h_{\varepsilon, k+1}^{i}(x, t)=\sum_{j \neq i} R_{\varepsilon}^{i i} * h_{\varepsilon, k}^{j}(x, t) .
\end{gathered}
$$

Starting with

$$
f_{\varepsilon, 0}^{i}(x, t)=f_{\varepsilon}^{i}(x, t)
$$

we similarly define the sequence of functions $f_{\varepsilon, k}^{i}(x, t)$. It is clear that

$$
f_{\varepsilon}^{i}(x, t)=\sum_{k=0}^{N-1} h_{\varepsilon, k}^{i}(x, t)+f_{\varepsilon, N}^{i}(x, t)
$$

for all natural numbers $N$. Fix an arbitrary number $\delta>0$ and choose a natural number $N=N(\delta)$ so large that

$$
\sup _{i} \sup _{x \in E} \sup _{t \geq 0} f_{\varepsilon, N}^{i}(x, t)<\delta
$$


for all sufficiently small $\varepsilon>0$. Such a number $N$ exists in view of condition (18). In what follows we need the following auxiliary result.

Fix $\alpha \geq 0, i \neq j$, and put

$$
\begin{aligned}
& \widehat{Q}_{\varepsilon, \alpha}^{i}(x, A)=\int_{0}^{\infty} e^{-\alpha s / t} Q_{\varepsilon}^{i}(x, A \times d s), \\
& \widehat{Q}_{\varepsilon, \alpha}^{i j}(x, A)=\int_{0}^{\infty} e^{-\alpha s / t} Q_{\varepsilon}^{i j}(x, A \times d s), \\
& \widehat{H}_{\varepsilon, \alpha}^{i}(x, A)=\int_{0}^{\infty} e^{-\alpha s / t} H_{\varepsilon}^{i}(x, A \times d s), \\
& \widehat{R}_{\varepsilon}^{i j}(x, A)=\int_{0}^{\infty} e^{-\alpha s / t} R_{\varepsilon}^{i j}(x, A \times d s) .
\end{aligned}
$$

It is clear that

$$
\begin{gathered}
\widehat{Q}_{\varepsilon}^{i}(x, A) \leq \pi_{D}(A), \\
\lim _{\varepsilon \rightarrow 0} \widehat{Q}_{\varepsilon, \alpha}^{i}(x, A)=\pi_{D}(A)
\end{gathered}
$$

uniformly in $x \in D, A \in \mathcal{B}$.

Denote by $\lambda_{\varepsilon, \alpha}^{i}$ the Perronian root of the kernel $\widehat{Q}_{\varepsilon, \alpha}^{i}$, and by $e_{\varepsilon, \alpha}^{i}(x)$ and $\rho_{\varepsilon, \alpha}^{i}(A)$ the eigenfunction and eigenmeasure, respectively, that correspond to the Perronian root $\lambda_{\varepsilon, \alpha}^{i}$, that is,

$$
\begin{aligned}
& \int_{D} \widehat{Q}_{\varepsilon, \alpha}^{i}(x, d y) e_{\varepsilon, \alpha}^{i}(y)=\lambda_{\varepsilon, \alpha}^{i} e_{\varepsilon, \alpha}^{i}(x), \quad x \in D, \\
& \int_{D} \rho_{\varepsilon, \alpha}^{i}(d x) \widehat{Q}_{\varepsilon, \alpha}^{i}(x, A)=\lambda_{\varepsilon, \alpha}^{i} \rho_{\varepsilon, \alpha}^{i}(A),
\end{aligned}
$$

For the sake of brevity we put

$$
e_{\varepsilon}^{i}(x)=e_{\varepsilon, 0}^{i}(x), \quad \rho_{\varepsilon}^{i}(A)=\rho_{\varepsilon, 0}^{i}(A)
$$

and assume that

$$
\begin{gathered}
\rho_{\varepsilon}^{i}(D)=1, \quad \int_{D} \rho_{\varepsilon}^{i}(d x) e_{\varepsilon}^{i}(x)=1 \\
\int_{D} \rho_{\varepsilon, \alpha}^{i}(d x) e_{\varepsilon}^{i}(x)=1, \quad \alpha>0 \\
\int_{D} \rho_{\varepsilon, \alpha}^{i}(d x) e_{\varepsilon, \alpha}^{i}(x)=1, \quad \alpha>0 .
\end{gathered}
$$

Equality (23) and the theorem on the perturbation of separated parts of the spectrum [3. Chapter 4, $\S 4$, Theorem 3.16] imply that

$$
\begin{gathered}
\lambda_{\varepsilon, \alpha}^{i} \rightarrow 1, \\
e_{\varepsilon, \alpha}^{i}(x) \rightarrow 1
\end{gathered}
$$

uniformly in $x \in D$, and

$$
\rho_{\varepsilon, \alpha}^{i}(A) \underset{\varepsilon \rightarrow 0}{\longrightarrow} \pi_{D}(A)
$$

uniformly in $A \in \mathcal{B}$ for all $\alpha \geq 0$.

Finally we put

$$
\rho_{\varepsilon}^{i j}(A)=\frac{1}{1-\lambda_{\varepsilon, 0}^{i}} \rho_{\varepsilon}^{i} Q_{\varepsilon}^{i j}(A)=\frac{1}{1-\lambda_{\varepsilon, 0}^{i}} \int_{D} \rho_{\varepsilon}^{i}(d x) Q_{\varepsilon}^{i j}(x, A)
$$

for $i \neq j$, where

$$
Q_{\varepsilon}^{i j}(x, A)=\widehat{Q}_{\varepsilon, 0}^{i j}(x, A)=\mathbb{P}_{x, i}\left\{X(\tau) \in A, \xi_{\varepsilon}(\tau)=j\right\} .
$$


Lemma 1. If all the assumptions of Theorem 1 hold, then

$$
\sum_{j \neq i}\left|\widehat{R}_{\varepsilon, \alpha}^{i i}(x, A)-\frac{c_{i i}}{c_{i i} u-\alpha} \rho_{\varepsilon}^{i j}(A)\right| \underset{\substack{t \rightarrow \infty \\ \varepsilon \rightarrow 0 \\ \varepsilon t \rightarrow u}}{\longrightarrow} 0
$$

for all $u \geq 0$ and $\alpha \geq 0$ uniformly in $x \in D, A \in \mathcal{B}$.

Proof. The kernels $\widehat{Q}_{\varepsilon, \alpha}^{i}, \widehat{Q}_{\varepsilon, \alpha}^{i j}, \widehat{H}_{\varepsilon, \alpha}^{i}$, and $\widehat{R}_{\varepsilon, \alpha}^{i}$ are such that

$$
\begin{gathered}
\widehat{H}_{\varepsilon, \alpha}^{i}=\left(1-Q_{\varepsilon, \alpha}^{i}\right)^{-1}, \\
\widehat{R}_{\varepsilon, \alpha}^{i j}=\widehat{H}_{\varepsilon, \alpha}^{i} \widehat{Q}_{\varepsilon, \alpha}^{i j} .
\end{gathered}
$$

It is also clear that

$$
\begin{aligned}
& \widehat{Q}_{\varepsilon, \alpha}^{i}(x, A)=\mathbb{P}_{x, i}\left[e^{-\alpha \tau / t}, X(\tau) \in A, \xi_{\varepsilon}(\tau)=i\right], \\
& \widehat{Q}_{\varepsilon, \alpha}^{i j}(x, A)=\mathbb{P}_{x, i}\left[e^{-\alpha \tau / t}, X(\tau) \in A, \xi_{\varepsilon}(\tau)=j\right] .
\end{aligned}
$$

According to the theorem on the isolated eigenvalue,

$$
\widehat{H}_{\varepsilon, \alpha}^{i}(x, A)-\frac{1}{1-\lambda_{\varepsilon, \alpha}^{i}} e_{\varepsilon, \alpha}^{i}(x) \rho_{\varepsilon, \alpha}^{i}(A) \underset{\varepsilon \rightarrow 0}{\longrightarrow} I(x, A)-\pi_{D}(A)
$$

uniformly in $x \in D$ and $A \in \mathcal{B}$ (see [1, Chapter 3, $\S 3$, Theorem 1]). This together with (23) implies that

$$
\sum_{j \neq i}\left[\widehat{R}_{\varepsilon, \alpha}^{i j}(x, A)-\frac{m \varepsilon}{1-\lambda_{\varepsilon, \alpha}^{i}} e_{\varepsilon, \alpha}^{i}(x) \rho_{\varepsilon, \alpha}^{i j}(A)\right] \underset{\varepsilon \rightarrow 0}{\longrightarrow} 0
$$

uniformly in $x \in D$ and $A \in \mathcal{B}$, where

$$
\rho_{\varepsilon, \alpha}^{i j}(A)=\frac{1}{m \varepsilon} \rho_{\varepsilon, \alpha}^{i} Q_{\varepsilon, \alpha}^{i j}(A) .
$$

Now relations (22) and (23) imply that

$$
1-\lambda_{\varepsilon, \alpha}^{i} \sim 1-\mathbb{P}_{\pi_{D}, i}\left[e^{-\alpha \tau / t}, \xi_{\varepsilon}(\tau)=i\right]
$$

as $\varepsilon \rightarrow 0$. Applying the latter relation for $\alpha=0$, we obtain from (10) that

$$
\frac{1-\lambda_{\varepsilon, 0}^{i}}{\varepsilon} \underset{\varepsilon \rightarrow 0}{\longrightarrow}-m c_{i i} \text {. }
$$

The right hand side of (27) for $\alpha>0$ is equal to

$$
1-\mathbb{P}_{\pi_{D}, i}\left\{\xi_{\varepsilon}(\tau)=i\right\}+\mathbb{P}_{\pi_{D}, i}\left[\left(1-e^{-\alpha \tau / t}\right), \xi_{\varepsilon}(\tau)=i\right] .
$$

The second term in (29) divided by $\alpha / t$ approaches $m$ as $t \rightarrow \infty$. Thus the whole expression (29) equals

$$
1-\mathbb{P}_{\pi_{D}, i}\left\{\xi_{\varepsilon}(\tau)=i\right\}+m \frac{\alpha}{t}+o\left(\frac{1}{t}\right) .
$$

Using equality (10) we evaluate the limit:

$$
\lim _{\substack{t \rightarrow \infty \\ \varepsilon \rightarrow 0 \\ \varepsilon t \rightarrow u}} t\left[1-\mathbb{P}_{\pi_{D}, i}\left\{\xi_{\varepsilon}(\tau)=i\right\}+m \frac{\alpha}{t}+o\left(\frac{1}{t}\right)\right]=m\left(-c_{i i} u+\alpha\right),
$$

whence

$$
t\left(1-\lambda_{\varepsilon, \alpha}^{i}\right) \underset{\substack{t \rightarrow \infty \\ \varepsilon \rightarrow 0 \\ \varepsilon t \rightarrow u}}{\longrightarrow} m\left(-c_{i i} u+\alpha\right)
$$


The next step is to prove that

$$
t\left[\rho_{\varepsilon, \alpha}^{i}(A)-\rho_{\varepsilon}^{i}(A)\right] \underset{\substack{\varepsilon \rightarrow 0 \\ t \rightarrow \infty}}{\longrightarrow} \alpha m \pi_{D}(A)-\alpha \mathbb{P}_{\pi_{D}}[\tau, X(\tau) \in A]
$$

uniformly in $A \in \mathcal{B}$.

By the definition of $\rho_{\varepsilon, \alpha}^{i}$ and $\rho_{\varepsilon}^{i}$ we have

$$
\left(\rho_{\varepsilon, \alpha}^{i}-\rho_{\varepsilon}^{i}\right)\left(Q_{\varepsilon}^{i}-\lambda_{\varepsilon}^{i}\right)=\rho_{\varepsilon, \alpha}^{i}\left(Q_{\varepsilon}^{i}-\widehat{Q}_{\varepsilon, \alpha}^{i}\right)+\left(\lambda_{\varepsilon, \alpha}^{i}-\lambda_{\varepsilon}^{i}\right) \rho_{\varepsilon, \alpha}^{i}
$$

where $Q_{\varepsilon}^{i}=\widehat{Q}_{\varepsilon, 0}^{i}$ and $\lambda_{\varepsilon}^{i}=\lambda_{\varepsilon, 0}^{i}$.

According to (31) and (28), we have

$$
t\left[\lambda_{\varepsilon, \alpha}^{i}-\lambda_{\varepsilon}^{i}\right] \underset{\substack{\varepsilon \rightarrow 0 \\ t \rightarrow \infty \\ \varepsilon t \rightarrow u}}{\longrightarrow}-m \alpha
$$

for $u>0$. Furthermore, put

$$
\bar{Q}_{\varepsilon}^{i}(x, A)=\mathbb{P}_{x, i}\left[\tau, X(\tau) \in A, \xi_{\varepsilon}(\tau)=i\right] .
$$

Since

we get

$$
Q_{\varepsilon}^{i}(x, A)-\widehat{Q}_{\varepsilon, \alpha}^{i}(x, A)=\mathbb{P}_{x, i}\left[\left(1-e^{-\alpha \tau / t}\right), X(\tau) \in A, \xi_{\varepsilon}(\tau)=i\right]
$$

$$
\begin{aligned}
0 & \leq \alpha \bar{Q}_{\varepsilon}^{i}(x, A)-t\left[Q_{\varepsilon}^{i}(x, A)-\widehat{Q}_{\varepsilon, \alpha}^{i}(x, A)\right] \\
& \leq \mathbb{P}_{x, i}\left[\left(\alpha \tau-\frac{1-e^{-\alpha \tau / t}}{\alpha / t}\right), X(\tau) \in A, \xi_{\varepsilon}(\tau)=i\right] \\
& \leq \mathbb{P}_{x, i}\left(\alpha \tau-\frac{1-e^{-\alpha \tau / t}}{\alpha / t}\right) .
\end{aligned}
$$

In view of (7), the last expression approaches zero uniformly in $x \in D$ as $t \rightarrow \infty$. Moreover, (6) and (7) imply

$$
\bar{Q}_{\varepsilon}^{i}(x, A) \underset{\varepsilon \rightarrow 0}{\longrightarrow} \bar{Q}(x, A)=\mathbb{P}_{x}[\tau, X(\tau) \in A]
$$

uniformly in $x \in D$ and $A \in \mathcal{B}$. Thus,

$$
t\left[Q_{\varepsilon}^{i}(x, A)-\widehat{Q}_{\varepsilon, \alpha}^{i}(x, A)\right] \underset{\substack{t \rightarrow \infty \\ \varepsilon \rightarrow 0}}{\longrightarrow} \alpha \bar{Q}(x, A)
$$

uniformly in $x \in D$ and $A \in \mathcal{B}$.

Furthermore, let $V_{\varepsilon}$ be the generalized inverse operator of $Q_{\varepsilon}^{i}-\lambda_{\varepsilon}^{i}$; that is,

$$
V_{\varepsilon}=\frac{1}{2 \pi i} \oint\left(z-Q_{\varepsilon}^{i}\right)^{-1} \frac{d z}{z-\lambda_{\varepsilon}^{i}},
$$

where $C=\left\{z \in \mathbb{C}:|z|=\frac{1}{3}\right\}$ is the circle of radius $\frac{1}{3}$ in the complex plane centered at the origin. Since $\lambda_{\varepsilon}^{i}>\frac{1}{3}$ for sufficiently small $\varepsilon$, the operator $V_{\varepsilon}$ is well defined. Therefore

$$
\left(Q_{\varepsilon}^{i}-\lambda_{\varepsilon}^{i}\right) V_{\varepsilon}(x, A)=I(x, A)-e_{\varepsilon}(x) \rho_{\varepsilon}(A) .
$$

It is also clear that

$$
V_{\varepsilon}(x, A) \underset{\varepsilon \rightarrow 0}{\longrightarrow} \pi_{D}(A)-I(x, A)
$$

uniformly in $x \in D$ and $A \in \mathcal{B}$.

Using (24) and (25) we obtain from (33) and (37) that

$$
t\left[\rho_{\varepsilon, \alpha}^{i}(A)-\rho_{\varepsilon}^{i}(A)\right]=t \rho_{\varepsilon, \alpha}^{i}\left(Q_{\varepsilon}^{i}-Q_{\varepsilon, \alpha}^{i}\right) V_{\varepsilon}(A)+t\left(\lambda_{\varepsilon, \alpha}^{i}-\lambda_{\varepsilon}^{i}\right) \rho_{\varepsilon, \alpha}^{i} V_{\varepsilon}(A) .
$$

Combining (35), (34), and (38) and collecting the like terms we prove (32). 
We see from (26), (31), and (32) that the lemma follows from

$$
\sup _{x \in D} \sup _{A \in \mathcal{B}} \sum_{j \neq i} t\left|Q_{\varepsilon}^{i j}(x, A)-\widehat{Q}_{\varepsilon, \alpha}^{i j}(x, A)\right| \underset{\substack{\varepsilon \rightarrow 0 \\ t \rightarrow \infty}}{\longrightarrow} 0
$$

and

$$
\sup _{\varepsilon>0} \sum_{j \neq i} \rho_{\varepsilon}^{i j}(D)<\infty .
$$

We start with the proof of (39). The sum in (39) is nonnegative and does not exceed

$$
\sum_{j \neq i} t \mathbb{P}_{x, i}\left[\left(1-e^{-\alpha \tau / t}\right), \xi_{\varepsilon}(\tau)=j\right]
$$

In turn, the last expression does not exceed

$$
\alpha \mathbb{P}_{x}[\tau, \tau>T]+\alpha T\left[1-\mathbb{P}_{x, i}\left\{\xi_{\varepsilon}(\tau)=i\right\}\right]
$$

for any $T>0$. The first term in the last expression approaches zero uniformly in $x \in D$ as $T \rightarrow \infty$ in view of (14), while the second term, for every fixed $T>0$, approaches zero uniformly in $x \in D$ as $\varepsilon \rightarrow 0$. Relation (39) is proved.

To prove (40) note that

$$
\begin{aligned}
\sum_{j \neq i} \rho_{\varepsilon}^{i j}(D) & \leq \frac{1}{1-\lambda_{\varepsilon}^{i}} \int_{D} \rho_{\varepsilon}^{i}(d x)\left[1-\mathbb{P}_{x, i}\left\{\xi_{\varepsilon}(\tau)=i\right\}\right] \\
& =\frac{1}{1-\lambda_{\varepsilon}^{i}}\left[\rho_{\varepsilon}^{i}(D)-\rho_{\varepsilon}^{i} Q_{\varepsilon}^{i}(D)\right]=1 .
\end{aligned}
$$

In fact, we obtained a stronger result, namely

$$
\limsup _{\varepsilon \rightarrow 0} \sum_{j \neq i} \rho_{\varepsilon}^{i j}(D) \leq 1
$$

The lemma is proved.

Lemma 2. Suppose the assumptions of Lemma 1 hold and a bounded function $\Psi(x, t)$ is measurable with respect to the variables $x \in D$ and $t \geq 0$. We also assume that

$$
\sup _{x \in D}|\Psi(x, t+s)-\Psi(x, t)| \underset{s \rightarrow 0}{\longrightarrow} 0
$$

for all $t \geq 0$. Then

$$
\sum_{j \neq i}\left|\int_{D} \int_{0}^{t} R_{\varepsilon}^{i j}(x, d y \times t d s) \Psi(y, s)-c_{i j} u \int_{0}^{t} e^{c_{i i} u s} \Psi(y, s) d s\right| \underset{\substack{\varepsilon \rightarrow 0 \\ t \rightarrow \infty \\ \varepsilon t \rightarrow u}}{\longrightarrow} 0
$$

uniformly in $x \in D$ and $0 \leq t \leq T$, where $T>0, u>0$, and $i \in I$ are arbitrary.

Proof. Note that relation (42) holds uniformly in $t$ belonging to an arbitrary finite interval in view of compactness, that is,

$$
\sup _{t \in[0, T]} \sup _{x \in D}|\Psi(x, t+s)-\Psi(x, t)| \underset{s \rightarrow 0}{\longrightarrow} 0
$$

for all $T>0$. Lemma 1 and the continuity theorem for the Laplace transform imply

$$
\sum_{j \neq i}\left|\int_{D} \int_{0}^{t} R_{\varepsilon}^{i j}\left(x, d y \times\left[t s_{1}, t s_{2}\right)\right) \Psi(y)-\int_{D} \rho_{\varepsilon}^{i j}(d y) \Psi(y) \int_{s_{1}}^{s_{2}}\left(-c_{i i}\right) u e^{c_{i i} u s} d s\right| \underset{\substack{\varepsilon \rightarrow 0 \\ t \rightarrow \infty \\ \varepsilon t \rightarrow u}}{\longrightarrow} 0
$$

for all $0 \leq s_{1} \leq s_{2}$ and all bounded $\mathcal{B}$-measurable functions $\Psi(y)$. 
For a fixed $\delta>0$, put

$$
\begin{array}{ll}
\Psi_{\delta}^{+}(x, t)=\sup _{k \delta \leq s \leq k \delta+\delta} \Psi(x, s), & k \delta \leq t \leq k \delta+\delta, \\
\Psi_{\delta}^{-}(x, t)=\inf _{k \delta \leq s \leq k \delta+\delta} \Psi(x, s), & k \delta \leq t \leq k \delta+\delta .
\end{array}
$$

Since the function $\Psi(x, t)$ is continuous in $t$, the functions $\Psi_{\delta}^{ \pm}(x, t)$ are measurable with respect to all their arguments. Relation (44) implies that for all $T>0$,

$$
\sup _{t \in[0, T]} \sup _{x \in D}\left|\Psi_{\delta}^{ \pm}(x, t)-\Psi(x, t)\right| \underset{\delta \rightarrow 0}{\longrightarrow} 0 .
$$

Now relation (45) implies that

$$
\sum_{j \neq i}\left|\int_{D} \int_{0}^{t} R_{\varepsilon}^{i j}(x, d y \times t d s) \Psi_{\delta}^{ \pm}(y, s)-\int_{D} \rho_{\varepsilon}^{i j}(d y) \int_{0}^{t}\left(-c_{i i}\right) u e^{c_{i i} u s} \Psi_{\delta}^{ \pm}(y, s) d s\right| \underset{\substack{\varepsilon \rightarrow 0 \\ t \rightarrow \infty \\ \varepsilon t \rightarrow u}}{\longrightarrow} 0
$$

for all $\delta>0$. The limit

$$
\sum_{j \neq i}\left|\rho_{\varepsilon}^{i j}(D)-\frac{c_{i j}}{\left(-c_{i i}\right)}\right| \underset{\varepsilon \rightarrow 0}{\longrightarrow} 0
$$

exists by (10) and (11). Hence

$$
\sum_{j \neq i}\left|\int_{D} \int_{0}^{t} R_{\varepsilon}^{i j}(x, d y+t d s) \Psi_{\delta}^{ \pm}(y, s)-c_{i j} u \int_{0}^{t} e^{c_{i i} u s} \Psi_{\delta}^{ \pm}(y, s) d s\right| \underset{\substack{\varepsilon \rightarrow 0 \\ t \rightarrow \infty \\ \varepsilon \rightarrow u}}{\longrightarrow} 0 .
$$

This together with (46) implies (43). The lemma is proved.

\section{BIBLIOGRAPHY}

1. V. M. Shurenkov, Ergodic Theorems and Related Problems, "Nauka", Moscow, 1989; English transl., VSP International Science Publishers, Leiden, 1998. MR1690361 (2000i:60002)

2. N. I. Portenko, A. S. Skorokhod, and V. M. Shurenkov, Markov Processes, Progress in Science and Technology. Current Problems in Mathematics. Fundamental Directions, vol. 46, Vsesoyuz. Inst. Nauchn. i Tekhn. Inform., Akad. Nauk SSSR, Moscow, 1989. (Russian) MR1039619 (91j:60113b)

3. T. Kato, Perturbation Theory for Linear Operators, Springer-Verlag, Berlin-New York, 1966. MR0203473 (34:3324)

Department of Higher Mathematics, Vadym Hetman Kyiv National Economic University, Peremogy Avenue 54/1, Kyiv 03057, Ukraine

E-mail address: dsv@bms.com.ua

Received 6/SEP/2005

Translated by OLEG KLESOV 